\title{
Texture analysis of strongly oriented MAPbI3 layers using fast reciprocal-space mapping
}

\author{
Lukáš Horák ${ }^{1}$, Amalraj Peter Amalathas², Jakub Holovský,3 \\ ${ }^{1}$ Charles University, Faculty of Mathematics and Physics, Prague, Czech Republic; \\ ${ }^{2}$ Centre for Advanced Photovoltaics, Faculty of Electrical Engineering, Czech Technical University in Prague, 16627 Prague, Czech \\ Republic; \\ ${ }^{3}$ Institute of Physics, Czech Academy of Sciences, 16200 Prague, Czech Republic \\ horak@karlov.mff.cuni.cz
}

Polycrystalline layers of organic perovskites such as $\mathrm{CH} 3 \mathrm{NH} 3 \mathrm{PbI} 3$ (MAPbI3) are intensively studied in order to achieve high performance of solar cells. The final efficiency correlates with the defect density, size and morphology of the crystallites. These materials exhibit tendency to form strongly oriented layers with sharp fibre multi-component texture. In order to correlate the relevant physical properties of the layers with their crystallographic orientation, it is highly desirable to easily measure the present texture.

As a classical and well-established approach, one can use pole figure measurement to fully determine the texture of the layers, however here the task is complicated by the fact that the low-symmetry unit cell of this material is rather large. Consequently, the number of observable peaks is high and their diffraction angles are partially overlapping. In order to resolve them, the resolution in diffraction angle has to be increased at the expense of the intensity making the pole figure measurement to be a time consuming. Another specific problem can be a presence of some possible strain influencing the peak positions.

Fortunately, fast 2D detectors are more and more available also in standard laboratory diffractometers that makes it possible to measure reciprocal-space maps very quickly. In this presentation, the measurements with $2 \mathrm{D}$ detector placed closely behind the sample are presented. Using the shorter sample-detector distance, the resolution is partially sacrificed while the reciprocal space area observed by the detector is dramatically extended. In this configuration, the continuous theta-2theta scan fully probes a long stripe in a reciprocal space. By measuring several such stripes, it is easy to reveal the full planar cut of the reciprocal space, and surprisingly the total acquisition time can be only tens of minutes for strongly oriented layers. Moreover, such measurement can be performed for different sample azimuth in order to obtain different planar cuts. This is desirable for single-crystal substrates, for which the surface symmetry can be followed.

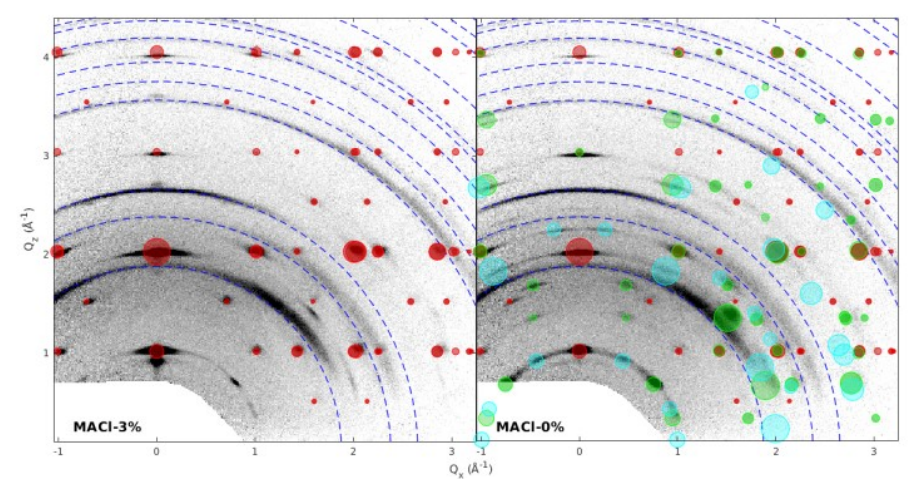

Figure 1. Wide Reciprocal Space Maps for the $\mathrm{MAPbI}_{3}$ thin layers grown with the presence of MACl additive. The concentration of $\mathrm{MACl}$ during the growth determines the final texture of the layer. A low concentration results in a multi-component texture whereas a high concentration enhances just one preferential orientation. Transparent spots represent simulated peak positions for the same

$\mathrm{MAPbI}_{3}$ structure, the texture components are resolved by different colours.

The obvious advantage of this approach is a possibility to quickly visualise the intensity in reciprocal space and to compare the obtained images with the simulations based on some expected phase/texture model giving semi-quantitative results. Therefore it is very suitable for the first-try characterization of the unknown samples.

\section{Keywords: perovskite solar cells, texture, methylammonium lead halide}

The work was supported by the project NanoCent financed by European Regional Development Fund (ERDF, project No. CZ.02.1.01/0.0/0.0/15.003/0000485) 